the tail of the donor ground-state wavefunction, Patanè and co-workers were able to identify two regimes dominated by Coulomb and magnetic confinement, respectively. Lei and co-workers studied the shaping effect of the field on two orbitals of the quantum-dot $p$-shell (Fig. 2). At zero field, one orbital has $x$-like symmetry and the other $y$-like symmetry, reflecting the elliptical anisotropy of the dot potential. With increasing field, they transform into rings, consistent with the circular symmetry of cyclotron orbits.

The above measurements do have one serious drawback, as they are averages over thousands of objects. Ideally, one would image a single artificial atom and manipulate it with various perturbations in addition to the magnetic field. A more fundamental issue is the role of electron-electron interaction, as several electrons populated the quantum dots studied by Lei and colleagues. In their case, the images could be understood in terms of non-interacting physics, but then what happens for dots filled by strongly interacting electrons ${ }^{9}$ ? A theory ${ }^{10}$ predicts that electron correlation may strongly reshape the wavefunction with respect to its uncorrelated counterpart. No doubt there is much to be learnt as more images are seen.
Massimo Rontani is at the Research Center on nanoStructures and bioSystems at Surfaces (S3) of CNR - Istituto Nanoscienze, Via Campi 213a, 41125 Modena, Italy.

e-mail:massimo.rontani@cnr.it

\section{References}

1. Loss, D. \& DiVincenzo, D. P. Phys. Rev. A 57, 120-126 (1998).

2. Patanè, A. et al. Phys. Rev. Lett. 105, 236804 (2010).

3. Lei, W. et al. Phys. Rev. Lett. 105, 176804 (2010).

4. Kane, B. E. Nature 393, 133-137 (1998).

5. Bardeen, J. Phys. Rev. Lett. 6, 57-59 (1961)

6. Sakai, J-W. et al. Phys. Rev, B 48, 5664-5667 (1993).

7. Jarosik, N. C. et al. Phys. Rev. Lett. 54, 1283-1286 (1985).

8. Sikorski, C. \& Merkt, U. Phys. Rev. Lett. 62, 2164-2167 (1989).

9. Kalliakos, S. et al. Nature Phys. 4, 467-471 (2008).

10. Rontani, M. \& Molinari, E. Phys. Rev. B 71, 233106 (2005).

11. Patanè, A. et al. Phys. Rev. B 65, 16538 (2002).

\title{
THE SMALLEST METALS
}

What is a metal? It depends on who you ask, for like most colloquial words used in science it has meant different things at different times. The etymology from ancient Greek probably refers to any substance mined or quarried. Most folks would expect a metal to be hard and silveryshiny; some sophisticates may demand electrical conductivity. Some will refer you to the periodic table; physicists may expect a partially filled electron band. (No one else, however, has yet come up with a definition quite as eccentric as that used by astronomers.)

But studies of atomic clusters reveal that the question 'metal or not?' depends not just on chemical composition but on size. The whole concept of electronic bands, on which discussions of metallic behaviour generally rest, is contingent on the overlap of a sufficient number of atomic orbitals to create a continuum of energy states. Or is it? A study by Bowlan et al. suggests that even the smallest sodium clusters can be considered to exhibit metallic behaviour ${ }^{1}$.

Quantum calculations and many experiments have seemed to imply a transition from molecular-like clusters to truly metallic particles as the number of atoms increases for elements that are bulk metals. Just a few examples: early work on mercury clusters $\mathrm{Hg}_{n}$ suggested an onset of metallicity above about $n=40$ (ref. 2); Mossbauer spectroscopy shows that the inner core atoms of clusters of about 55 gold atoms have a different charge density from those of bulk gold ${ }^{3}$; and photoelectron spectroscopy of magnesium clusters $\mathrm{Mg}_{n}$ shows bandgap closure at around $n=18$ (ref. 4).

Such findings raise questions about whether the popular, theoretically tractable jellium model is a good description of the smallest clusters of metal atoms. This more or less insists on regarding the cluster as a metal: a spherical droplet in which the nuclei and core electrons are embedded in a delocalized blob of valence electrons. Nonetheless, jellium seems a good approximation for predicting the electronic properties, such as polarizability ${ }^{5}$, even of some relatively small metal clusters.

Bowlan et al. now confront that picture with a particularly stringent test. Whereas earlier measurements of polarizability of sodium clusters were performed at temperatures where the clusters are liquid, they have made measurements at $20 \mathrm{~K}$, well below the freezing point.

They argue that the most general definition of a metal in terms of electronic behaviour is that a metallic object cannot sustain an internal electric field. The charge density will always rearrange to eliminate any inhomogeneity, meaning that there can be no electric dipole moment. Quantum-chemical calculations

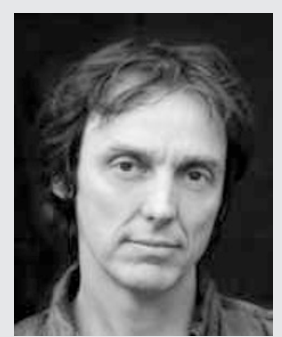

PHILIP BALL

suggest that small sodium clusters should have a non-vanishing dipole moment caused by imperfect screening of the ion cores by valence electrons ${ }^{6}$. Yet the cryogenic molecular-beam experiments of Bowlan et al. show that, not only are the polarizabilities smaller than reported in previous experiments, but the dipole moments are orders of magnitude smaller too and in fact very close to zero for all clusters, even dimers and trimers. On this measure, at least, sodium is a metal all the way down.

References

1. Bowlan, J., Liang, A. \& de Heer, W. A. Phys. Rev. Lett. 106, 043401 (2011).

2. Bréchignac, C. et al. Phys. Rev. Lett. 60, 275-278 (1988).

3. Mulder, F. M., Thiel, R. C., de Jongh, L. J. \& Gubbens, P. C. M. Nanostruct. Mater. 7, 269-292 (1996).

4. Thomas, O. C., Zheng, W., Xu, S. \& Bowen, K. H. Jr Phys. Rev. Lett. 89, 213403 (2002).

5. Knight, W. D., Clemenger, K., de Heer, W. A. \& Saunders, W. A. Phys. Rev. B 31, 2539-2540 (1985).

6. Solovyov, I. A., Solovyov, A. V. \& Greiner, W. Phys. Rev. A 65, 053203 (2002). 